\title{
UHPLC-MS-MS analysis of oxylipins metabolomics components of follicular fluid in infertile individuals with diminished ovarian reserve
}

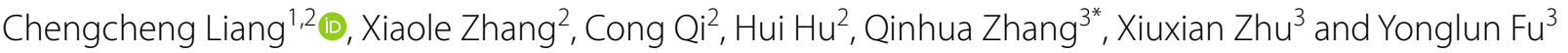

\begin{abstract}
Background: Diminished ovarian reserve (DOR) refers to a decrease in the number and quality of oocytes in the ovary, which results in a lack of sex hormones and a decline of fertility in women. DOR can potentially progress to premature ovarian failure (POF), which has a negative impact on women's quality of life and is a major cause of female infertility. Oxidative stress is a major contributor to fertility decrease in DOR patients, affecting the follicular microenvironment, oocyte maturation, fertilization, and embryo development. Understanding intracellular signal transduction can be achieved by defining specific oxidized lipid components in follicular fluid (FF) of DOR infertile patients.
\end{abstract}

Methods: The oxylipins metabolic signatures in the FF of DOR patients and females with normal ovarian reserve (NOR) enrolled for the in vitro fertilization (IVF) cycle were analyzed using UHPLC-MS-MS technology. Principal component analysis (PCA) and orthogonal projections to latent structure discriminant analysis (OPLS-DA) were used to analyze the derived metabolomic profiles. Pathway enrichment analysis was carried out using the Kyoto Encyclopedia of Genes and Genomes (KEGG) and MetaboAnalyst databases. Furthermore, the Spearman rank correlation coefficient was used to determine the correlation between age, FSH, AMH, AFC, oocytes retrieved, MIl oocytes, fertilization, highquality embryos, and the concentration of differential oxidized lipid metabolites in FF.

Results: Fifteen oxylipins metabolites were found to be lower in the FF of DOR patients than those in the NOR group,

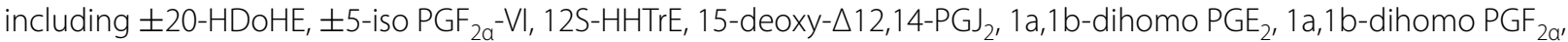
20-COOH-AA, 20-HETE, 8S,15S-DiHETE, $\mathrm{PGA}_{2}, \mathrm{PGD}_{2}, \mathrm{PGE}_{1}, \mathrm{PGF}_{1 \alpha^{\prime}} \mathrm{PGF}_{2 \alpha^{\prime}}$ and $\mathrm{PGJ} \mathrm{J}_{2}$. The pathway enrichment analysis revealed that the 15 differentially oxidized lipid metabolites were closely related to the arachidonic acid metabolic pathway. Correlation analysis revealed that the concentration of 8 different oxidized lipid metabolites in FF was negatively correlated to FSH and positively correlated with AFC. AMH, the number of oocytes retrieved, MII oocytes and fertilization, were all positively correlated with 9 different oxidized lipid metabolites, but only one metabolite was positively correlated with the number of high-quality embryos.

Conclusions: Metabolomic analysis of FF revealed that oxylipins metabolism disorders were closely related to ovarian reserve function. Among these oxylipins metabolites, arachidonic acid metabolism undergoes significant changes that may be related to oocyte development, resulting in decreased fertility in DOR patients.

\footnotetext{
*Correspondence: shumeier@163.com

${ }^{3}$ Shanghai First Maternity and Infant Hospital, Tongji University School

of Medicine, Shanghai 201204, China

Full list of author information is available at the end of the article
}

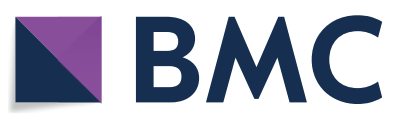

(c) The Author(s) 2021. Open Access This article is licensed under a Creative Commons Attribution 4.0 International License, which permits use, sharing, adaptation, distribution and reproduction in any medium or format, as long as you give appropriate credit to the original author(s) and the source, provide a link to the Creative Commons licence, and indicate if changes were made. The images or other third party material in this article are included in the article's Creative Commons licence, unless indicated otherwise in a credit line to the material. If material is not included in the article's Creative Commons licence and your intended use is not permitted by statutory regulation or exceeds the permitted use, you will need to obtain permission directly from the copyright holder. To view a copy of this licence, visit http://creativecommons.org/licenses/by/4.0/. The Creative Commons Public Domain Dedication waiver (http://creativeco mmons.org/publicdomain/zero/1.0/) applies to the data made available in this article, unless otherwise stated in a credit line to the data. 
Trial registration: ChiCTR, ChiCTR2000038182, Registered 12 September 2020-Retrospectively registered

Keywords: Diminished ovarian reserve, Follicular fluid, In vitro fertilization, Oxylipins, Metabolomics

\section{Introduction}

Diminished ovarian reserve (DOR) refers to a decrease in the number and quality of oocytes in the ovary, which leads to decreased female fertility and reproductive endocrine function disorders. The most common clinical manifestation of women under the age of 40 years is rare menstruation, amenorrhea, and infertility. If not treated promptly, it may progress to premature ovarian failure (POF) within 1 6 years [1]. The incidence of DOR in infertile women of different ages is estimated to range from $6 \%$ to $64 \%$ [2]. According to the US Center for Disease Control and Prevention's national assisted reproductive technology data from 2014, DOR patients accounted for about $30 \%$ of pregnant women [3]. Due to the reduction in basic antral follicle count (AFC), the reactivity of DOR patients to ovulation-promoting drugs decreased during in vitro fertilization /intracytoplasmic sperm injection-embryo transfer (IVF/ICSI-ET), resulting in some phenomena such as poor ovarian responder (POR), high cycle cancellation rate, decreased number of oocytes, decreased number of high-quality embryos, and decreased cumulative pregnancy rate [4].

Oxidative stress (OS) is the phenomenon in which excessive reactive oxygen species (ROS) are produced after being stimulated in vivo and in vitro, exceeding the antioxidant capacity in the body, and there is a serious imbalance between oxidation and antioxidant system in the body, resulting in oxidative stress damage to cells and tissues [5]. ROS primarily consists of superoxide anion $\left(\mathrm{O}_{2}^{-} \cdot\right)$, hydrogen peroxide $(\mathrm{H} 2 \mathrm{O} 2)$, hydroxyl radical $(\cdot \mathrm{OH})$, etc. Under physiological conditions in vivo, ROS acts as a key second messenger in intercellular signal transmission and modulates gene expression to maintain cell homeostasis. Excessive ROS accumulation in tissue cells, on the other hand, cause damage to proteins, nucleic acids, and lipids, resulting in the mutation or deletion of nuclear DNA and mitochondrial DNA, lipid peroxidation of cell membranes leading to changes in membrane fluidity, protein oxidative damage leading to the inactivation of important enzymes such as superoxide dismutase (SOD), catalase (CAT) and glutathione peroxidase (GSH-Px), and finally induce apoptosis and tissue structure damage [6]. The cell membrane, which is rich in polyunsaturated fatty acids, is a susceptible target of ROS attack. ROS reacts with phospholipids, enzymes, and membrane receptor-related macromolecules on the membrane surface to form malondialdehyde (MDA), 4-Hydroxynonenal, isomeric prostaglandins, and other active compounds, particularly lipid peroxides [7]. OS plays an important role in the physiological and pathological processes of the female reproductive system, which is one of the major reasons for the decline of female reproductive function. According to recent research, OS can cause ovarian endocrine dysfunction, oocyte quality decline, granulosa cell apoptosis, and then follicular atresia, which is an important reason for the decline in fertility of DOR patients, and has a significant impact on the follicular microenvironment, oocyte maturation, fertilization and embryo development [8-10].

Follicular fluid (FF) is the fluid in which oocytes exchange materials and metabolize energy with surrounding cells. Its metabolite composition can represent the level of the FF environment and, in turn, the level of oocyte metabolism. It is made up of secretions produced by peripheral granulosa cells and serum diffused by local capillaries, as well as plasma components that have crossed the blood follicular barrier, primarily hormones, growth factors, interleukins, anti-apoptosis factors, proteins, sugars, amino acids, active oxygen, and antioxidant enzymes [11, 12]. It has an impact on oocyte maturation, follicular wall rupture, fertilization, and the development of early embryos [13, 14]. Oxidative stress markers in FF are closely related to the growth, development, and maturation of oocytes. Infertility caused by DOR has long been an inescapable problem in reproductive medicine, and changes in associated metabolites in FF indicate the quality of oocytes. As a result, studying the oxylipins metabolomics in FF of infertile patients with DOR is useful.

Metabolomics, as an integral component of system biology, mimics the research ideas of genomics and proteomics, through quantitative analysis of metabolites in organisms, and determining the relative link between metabolites and physiological and pathological changes [15]. Metabolomics has recently been used to identify potential biomarkers in FF. The study of FF metabolomics is critical for evaluating and predicting the potential of in vitro fertilization and oocyte embryo development. Merhi et al. [16] used metabolomics to identify biomarkers of ovarian reserve function. The results showed that advanced glycation end-products (AGE) in FF were positively correlated with the level of anti-Mullerian hormone (AMH), and AGE could be used as an important biological index for predicting ovarian reserve in ART patients. Another metabolomics study found that the concentration of glucose in the FF of DOR 
patients decreased significantly, whereas the concentrations of lactate and progesterone increased significantly. At the same time, glucose uptake, lactic acid production, platelet-type phosphofructokinase gene expression in granulosa and cumulus cells increased significantly, while progesterone concentration decreased significantly, implying that decreased aerobic metabolism, increased anaerobic metabolism and high progesterone stimulation of DOR oocytes may be important reasons for their quality decline and early embryo dysplasia [17]. The metabolomics of oxylipins in FF of DOR patients, on the other hand, has not been documented.

In this study, UHPLC-MS-MS technology was used to detect oxylipins metabolites in FF of DOR infertile patients using target metabolomics, to identify the oxidized lipid metabolites in FF of DOR infertile patients, to screen potential biomarkers, and to investigate the possible mechanism of oocyte quality decline in DOR, which has far-reaching implications for understanding the pathogenesis of DOR.

\section{Materials and methods}

\section{Sample collection and preparation}

Patients with DOR $(n=20)$ and women with normal ovarian reserve (NOR) $(\mathrm{n}=20$, as a control group) were recruited from the Reproductive Medicine Center of Shanghai first maternity and infant hospital, Tongji university school of medicine, between May 2020 and January 2021. The study was approved by the Ethics Committees of Shuguang hospital which is affiliated with Shanghai University of traditional Chinese medicine (No.2020-833-40-01) and it was registered with the China clinical trial registration center (ChiCTR2000038182). Before the study, all subjects signed informed consent. DOR was diagnosed in patients based on the following diagnostic criteria [18]: (1) aged 20 40 years old. (2) requires one of the following three features to be met: 1) serum levels of basic follicle-stimulating hormone (FSH): 10 30 IU/L with or without FSH/ luteinizing hormone (LH) $\geq 3$; 2) serum levels of AMH: 20 25 years old $(\mathrm{AMH} \leq 3.0 \mathrm{ng} / \mathrm{ml}), 26 \sim 30$ years old $(\mathrm{AMH} \leq 2.5 \mathrm{ng} /$ $\mathrm{ml}), 31 \sim 35$ years old $(\mathrm{AMH} \leq 1.5 \mathrm{ng} / \mathrm{ml}), 36 \sim 40$ years old $(\mathrm{AMH} \leq 1.0 \mathrm{ng} / \mathrm{ml}) ; 3)$ basic AFC on menstrual cycle (MC) 2 3: AFC $\leq$ 5. DOR was diagnosed by meeting (1) and any two of (2). The control group included NOR patients who were infertile solely owing to tubal factors. The following were the detailed inclusion criteria: (1) aged 20 35 years old. (2) serum levels of basic FSH $<10 \mathrm{IU} / \mathrm{L}$, serum levels of basic $\mathrm{LH}<10 \mathrm{IU} / \mathrm{L}$, with FSH/ $\mathrm{LH}<3$. (3) the number of AFC: $8<\mathrm{AFC}<24$. (4) serum levels of AMH: 20 25 years old (3.0ng/ml<AMH $\leq 8.4 \mathrm{ng} /$ $\mathrm{ml}), 26 \sim 30$ years old $(2.5 \mathrm{ng} / \mathrm{ml}<\mathrm{AMH} \leq 8.4 \mathrm{ng} / \mathrm{ml}), 31 \sim 35$ years old $(1.5 \mathrm{ng} / \mathrm{ml}<\mathrm{AMH} \leq 8.4 \mathrm{ng} / \mathrm{ml})$.
The study exclusion criteria were as follows: (1) endometriosis. (2) diagnosis of the polycystic ovarian syndrome. (3) presence of a functional ovarian cyst with $E_{2}$ $>100 \mathrm{pg} / \mathrm{ml}$. (4) history of ovarian surgery such as ovarian cyst stripping and teratoma stripping. (5) receipt of hormone treatments within the preceding 3-month period. (6) any contraindications to ovarian stimulation treatment.

To avoid the influence of the ovulation induction scheme on the metabolites in FF in this study, all recruited patients, including DOR and NOR patients, adopted a micro-stimulation strategy for ovulation induction. Letrozole (Jiangsu Hengrui Medicine Co., China) was given orally for 5 days, beginning on MC 3, with a $5 \mathrm{mg}$ daily dose. We began ovarian stimulation with a daily injection of gonadotropin $(\mathrm{Gn})$ until the trigger day when the dominant follicular diameter of $\geq 10$ $\mathrm{mm}$ was confirmed by transvaginal ultrasonography after MC 6. The protocol for human menopausal gonadotropin (hMG) (Zhuhai Lizhu Pharmaceutical Trading Co., China) was used as reported previously [19]. hMG was injected intramuscularly daily in alternate doses of 150 and $225 \mathrm{IU}$ (150 IU of hMG given on the first day of ovarian stimulation, $225 \mathrm{IU}$ on the second day, $150 \mathrm{IU}$ on the third day, and so on). The hMG initiation dose was similar in the DOR and NOR groups. After 5 7 days, serum sex hormones [FSH, LH, estrogen $\left(\mathrm{E}_{2}\right)$, and progesterone $(\mathrm{P})]$ were detected, as well as a re-examination of the vaginal ultrasound. The hMG dosage was adjusted based on the level of sex hormones in the serum as well as the number and size of developing follicles. If more than one dominant follicle with a diameter $\geq 18 \mathrm{~mm}$ or more than three follicles with a diameter $\geq 16 \mathrm{~mm}$ were present, 3000 5000IU of human chorionic gonadotropin (hCG) (Zhuhai Lizhu Pharmaceutical Trading Co., China) was used to induce oocyte maturation. We used transvaginal ultrasound-guided follicle aspiration to retrieve oocytes 36 38 h after hCG administration. Following oocyte separation, FF obtained from 3 dominant follicles was pooled in a $15 \mathrm{ml}$ centrifuge tube and centrifuged at 3000 $\mathrm{rpm}$ for $15 \mathrm{~min}$ at $4^{\circ} \mathrm{C}$ to remove insoluble particles and cells. The supernatant was packaged in a $2 \mathrm{ml}$ freezing tube, and stored at $-80^{\circ} \mathrm{C}$ in an ultra-low temperature storage box to avoid repeated freezing or thawing.

\section{Serum hormone measurement and antral follicle calculation}

Serum sex hormones (FSH, $\mathrm{LH}, \mathrm{E}_{2}$, and $\mathrm{P}$ ) were detected in all patients by radio-immunoassay and transvaginal ultrasonography for basic AFC on MC 2 3. 


\section{Metabolites extraction}

A $1000 \mu \mathrm{L}$ aliquot of each sample was accurately transferred to an Eppendorf tube. Following the addition of the isotopically-labeled internal standard mixture, the samples were vortexed for $30 \mathrm{~s}$ and sonicated for $5 \mathrm{~min}$ in an ice-water bath. SPE was used to further purify the sample. The SPE cartridges were equilibrated with $1 \mathrm{~mL}$ of methanol and $1 \mathrm{~mL}$ of water. After loading a sample (supernatant obtained as mentioned above), the cartridge was washed with $1 \mathrm{~mL}$ of $5 \% \mathrm{MeOH} / \mathrm{H} 2 \mathrm{O}(\mathrm{v} / \mathrm{v})$. The flow-through fraction was then discarded. Finally, the samples were eluted with $1 \mathrm{~mL}$ of $\mathrm{MeOH}$, the eluent was evaporated to dryness under a gentle stream of nitrogen, and the eluent was reconstituted in $100 \mu \mathrm{L}$ of $30 \%$ $\mathrm{ACN} / \mathrm{H} 2 \mathrm{O}(\mathrm{v} / \mathrm{v})$. The clear supernatant was subjected to UHPLC-MS/MS analysis, after centrifugation (15 min, $\left.12000 \mathrm{rpm}, 4^{\circ} \mathrm{C}\right)$.

\section{UHPLC-MRM-MS analysis}

The UHPLC separation was performed using an EXIONLC System (Sciex), equipped with a Waters ACQUITY UPLC BEH C18 column $(150 \times 2.1 \mathrm{~mm}, 1.7$ $\mu \mathrm{m}$, Waters). The mobile phases A and B were $0.01 \%$ formic acid in the water, and $0.01 \%$ formic acid in acetonitrile, respectively. The temperature of the column was set to $50^{\circ} \mathrm{C}$. The temperature of the auto-sampler was set to $4^{\circ} \mathrm{C}$, and the injection volume was $10 \mu \mathrm{L}$.

For assay development, a SCIEX 6500 QTRAP+ triple quadrupole mass spectrometer (Sciex), equipped with an IonDrive Turbo V electrospray ionization (ESI) interface was used. Typical ion source parameters were as follows: Curtain Gas $=40$ psi, IonSpray Voltage $=-4500 \mathrm{~V}$, temperature $=500^{\circ} \mathrm{C}$, Ion Source Gas $1=30 \mathrm{psi}$, and Ion Source Gas $2=30$ psi.

Flow injection analysis was used to analyze the MRM parameters for each of the targeted analytes by injecting the standard solutions of the individual analytes, into the API source of the mass spectrometer. In the MRM scan mode, several most sensitive transitions were used to optimize the collision energy for each Q1/Q3 pair. The Q1/Q3 pairs with the highest sensitivity and selectivity among the optimized MRM transitions per analyte were selected as a "quantifier" for quantitative monitoring. The additional transitions served as a "quantifier" to confirm the identity of the target analytes. SCIEX Analyst Work Station Software (Version 1.6.3) and Multiquant 3.03 software (Version 20.2) were used for MRM data acquisition and processing.

\section{Data collection, processing and statistical analysis}

The data were statistically analyzed using SPSS 25.0 software. The baseline characteristics of the study population are described. The continuous variables with a normal or near-normal distribution were expressed as the means \pm standard deviations (SDs) and were analyzed by Student's t-test, otherwise, a Mann-Whitney U test was used. The frequency (composition ratio) of the classification data was statistically described and evaluated using the chisquare test. For correlation analyses, the Spearman rank correlation coefficient was used. Statistical significance was defined as $P<0.05$.

For the metabolomics analysis, the missing values in the raw data were filled by half of the minimum value. In addition, the overall normalization method was used for data analysis. SIMCA software (V16.0.2, Sartorius Stedim Data Analytics AB, Umea, Sweden) was used to perform multivariate analysis, principal component analysis (PCA), and orthogonal projections to latent structure discriminant analysis (OPLS-DA). The original data distribution was shown using PCA. OPLS-DA was used to examine the separation between two groups and to better understand the variables responsible for classification. The variable importance in the projection (VIP) of the first principal component obtained from the OPLSDA analysis was determined. In the univariate analysis, metabolites with a VIP $>1.0$ and $P$-value $<0.05$ were considered significantly different. Moreover, the quality of the OPLS-DA model was assessed using standard parameters $\left(R^{2} Y\right.$ and $\left.Q^{2}\right)$. The Kyoto Encyclopedia of Genes and Genomes (KEGG) (http://www.Genome.jp/kegg/) and the MetaboAnalyst 5.0 (https://www.metaboanalyst.ca/) database were used for pathway enrichment analysis.

\section{Results}

\section{Clinical characteristics and ovulation outcomes}

The average age, body mass index (BMI), duration of infertility, type of infertility, bAFC, AMH, bFSH, bLH, $\mathrm{bE}_{2}, \mathrm{P}$, the numbers of oocytes retrieved, MII oocytes, fertilization, and high-quality embryos of the participants are presented in Table 1 . There was no significant difference between DOR and NOR groups in terms of BMI, duration of infertility, type of infertility, bLH, bE2, and $\mathrm{P}$ levels $(P>0.05)$. The average age of the DOR group was significantly older than the NOR group $(P<0.05)$, bFSH levels in patients with DOR were significantly higher than in NOR patients $(P<0.05)$. However, bAFC, AMH, the number of retrieved oocytes, MII oocytes, fertilization, and high-quality embryos were significantly lower in DOR patients compared to NOR patients $(P<0.05)$. This indicated that the ovarian function of the DOR group was significantly low.

\section{Multivariate analysis of metabolites}

After relative standard deviation de-noising, 103 peaks were detected and 68 metabolites were identified in this study. The median vale was used to fill in the missing 
Table 1 The demographic and clinical characteristics of patients with DOR and NOR

\begin{tabular}{|c|c|c|c|}
\hline Item & DOR group $(n=20)$ & NOR group $(n=20)$ & $P$ value \\
\hline Age (year) & $35.350 \pm 4.017$ & $30.350 \pm 3.514$ & 0.001 \\
\hline $\mathrm{BMI}\left(\mathrm{kg} / \mathrm{m}^{2}\right)$ & $21.050 \pm 2.404$ & $20.876 \pm 1.780$ & 0.797 \\
\hline Duration of infertility(year) & $4.350 \pm 3.014$ & $2.900 \pm 1.586$ & 0.211 \\
\hline Type of infertility, n (\%) & & & 0.342 \\
\hline Primary & $9(45.00)$ & $11(55.00)$ & \\
\hline Secondary & $12(60.00)$ & $8(40.00)$ & \\
\hline $\mathrm{bFSH}(\mathrm{mlU} / \mathrm{ml})$ & $11.268 \pm 3.991$ & $6.331 \pm 1.084$ & 0.000 \\
\hline $\mathrm{bLH}(\mathrm{mlU} / \mathrm{ml})$ & $3.668 \pm 2.105$ & $3.783 \pm 1.151$ & 0.830 \\
\hline bE2 (pg/ml) & $55.025 \pm 43.448$ & $43.116 \pm 14.850$ & 0.529 \\
\hline $\mathrm{P}(\mathrm{ng} / \mathrm{ml})$ & $0.512 \pm 0.249$ & $0.569 \pm 0.163$ & 0.253 \\
\hline $\operatorname{bAFC}(n)$ & $2.850 \pm 1.461$ & $12.700 \pm 3.229$ & 0.000 \\
\hline $\mathrm{AMH}(\mathrm{ng} / \mathrm{mL})$ & $0.709 \pm 0.344$ & $3.900 \pm 1.189$ & 0.000 \\
\hline Oocytes retrieved (n) & $3.500 \pm 1.433$ & $14.200 \pm 4.884$ & 0.000 \\
\hline Mll oocytes (n) & $2.850 \pm 1.496$ & $10.350 \pm 4.146$ & 0.000 \\
\hline Fertilizations (n) & $2.750 \pm 1.410$ & $9.800 \pm 4.686$ & 0.000 \\
\hline High-quality embryos(n) & $0.750 \pm 0.786$ & $2.650 \pm 2.777$ & 0.012 \\
\hline
\end{tabular}

$D O R$ Diminished ovarian reserve, NOR Normal ovarian reserve, $B M I$ Body mass index, $b F S H$ basic follicle-stimulating hormone, $b L H$ basic luteinizing hormone, $b E_{2}$ basic estrogen, $P$ Progesterone, $b A F C$ basic antral follicle count, $A M H$ Anti-Mullerian hormone

variables. The final dataset, which included information on the peak number, sample name, and normalized peak area was imported into SIMCA software for multivariate analysis. To minimize the influence of both noise and high variance of the variables, the data were scaled and logarithmically transformed. Following these changes, PCA and OPLS-DA were used to comprehensively compare the FF metabolomic profiles and determine the degree of diversity between the DOR and NOR groups. Sixteen quality control (QC) samples were included to confirm the stability and repeatability of the system, and the QCs were clustered together and separated from the study subjects' samples, indicating that the PCA was correct (Fig. 1a). The PCA score plot revealed significant differences between DOR and NOR, with one outlier located beyond the 95\% Hotelling's T-squared ellipse (Fig. 1b). As shown in Fig. 1b, the majority of DOR samples clustered to the left, while all NOR samples clustered to the right. However, one DOR sample on the right was also visible in the PCA score plots.

An OPLS-DA was performed to compare the metabolite between DOR and NOR. The OPLS-DA score plot revealed that the oxidative lipid metabolites in FF differed significantly between DOR and NOR samples (Fig.1c). As shown in Fig.1c, all NOR samples clustered to the right, while the majority of DOR clustered to the left. However, there was one DOR sample located on the right. This finding suggested that there were substantial changes in oxidative lipid metabolites between DOR and NOR patients. Furthermore, the permutation test yielded
$\mathrm{R}^{2} \mathrm{Y}$ (cum) and $\mathrm{Q}^{2}$ (cum) values of 0.36 and -0.8 , respectively, indicating the lack of overfitting and the high predictive ability of the OPLS-DA model (Fig. 1d), and the suitability of the model for subsequent optimization analyses.

\section{Identification of significantly different metabolites and pathways associated with DOR}

In this study, 15 FF metabolites with VIP values $>1$ in the OPLS-DA analysis and $P$-values $<0.05$ in the univariate analysis were detected between DOR and NOR samples (Table 2), indicating that these 15 oxidized lipid metabolites were associated with ovarian reserve function. In particular, 15 metabolites were all reduced and no metabolites were increased in the DOR group compared to the NOR group. These 15 differentially oxidized lipid metabolites associated with ovarian reserve function were then summarized in a volcano plot (Fig. 2a): the 15 down-regulated metabolites (blue spots) were located on the left, while no up-regulated metabolites were found in the FF of DOR compared to the NOR group. Furthermore, a heatmap was used to distinguish the upregulated and downregulated metabolites in DOR patients compared with NOR patients (Fig. 2b). R software (Version 3.6.3) was also used to display the variations in 15 oxidized lipid metabolites in FF between the two groups. Among the differential metabolites, the levels of 15 differential metabolites were all lower in the DOR group than in the NOR control group (Fig.3). 

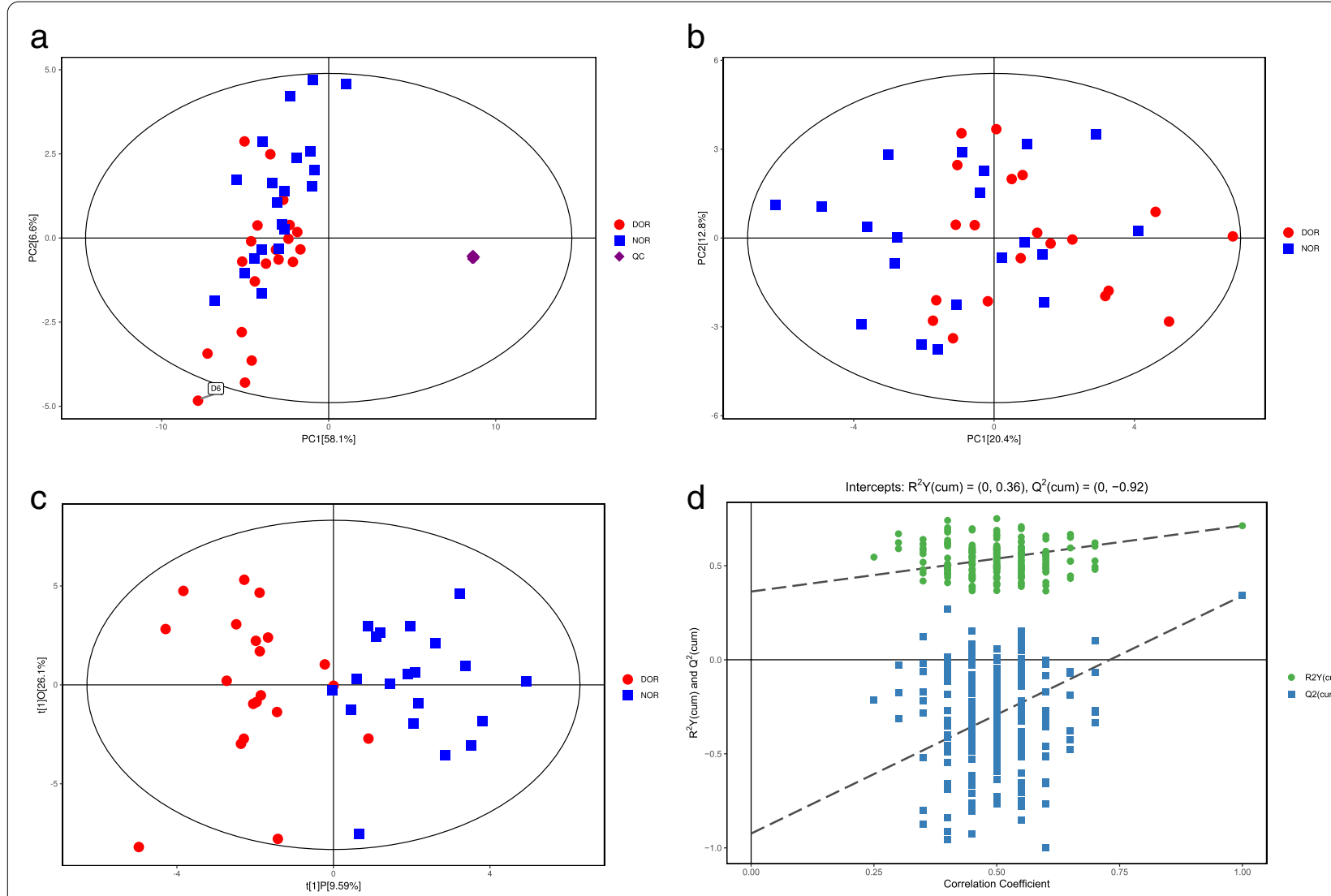

Fig. 1 PCA score plots, OPLS-DA score plots, and corresponding validation plot of OPLS-DA results derived from FF metabolomics profiles comparing DOR and NOR. a. PCA score plot with QC samples. b. PCA score plot without the QC samples. c. OPLS-DA score plot. d. The OPLS-DA model's permutation test

Table 2 Differential metabolites in FF samples between DOR and NOR groups

\begin{tabular}{|c|c|c|c|c|}
\hline Metabolites & VIP & $P$-value & Fold change & Type \\
\hline $\pm 20-\mathrm{HDoHE}$ & 1.46468 & 0.00481 & 0.68185 & Down \\
\hline \pm 5 -iso $\mathrm{PGF}_{2 a^{-} \mathrm{Vl}}$ & 1.05784 & 0.01656 & 0.55571 & Down \\
\hline 12S-HHTrE & 1.76019 & 0.00415 & 0.52086 & Down \\
\hline 15 -deoxy- $\triangle 12,14-P G J_{2}$ & 1.27131 & 0.03509 & 0.63420 & Down \\
\hline 1a,1b-dihomo $\mathrm{PGE}_{2}$ & 2.48940 & 0.01361 & 0.28674 & Down \\
\hline 1a,1b-dihomo $\mathrm{PGF}_{2 a}$ & 1.82901 & 0.02880 & 0.53937 & Down \\
\hline 20-COOH-AA & 1.75008 & 0.00401 & 0.67958 & Down \\
\hline 20-HETE & 1.11775 & 0.02031 & 0.29838 & Down \\
\hline 8S,15S-DiHETE & 1.38584 & 0.00450 & 0.70519 & Down \\
\hline $\mathrm{PGA}_{2}$ & 2.32409 & 0.01828 & 0.13424 & Down \\
\hline $\mathrm{PGD}_{2}$ & 1.08593 & 0.04879 & 0.36052 & Down \\
\hline $\mathrm{PGE}_{1}$ & 2.01038 & 0.00705 & 0.41340 & Down \\
\hline $\mathrm{PGF}_{1 \mathrm{a}}$ & 1.33950 & 0.04522 & 0.54329 & Down \\
\hline $\mathrm{PGF}_{2 a}$ & 2.18401 & 0.01426 & 0.34922 & Down \\
\hline$P G J_{2}$ & 1.66952 & 0.03160 & 0.24083 & Down \\
\hline
\end{tabular}

FF follicular fluid, DOR diminished ovarian reserve, NOR normal ovarian reserve, VIP variable importance in the projection
KEGG pathway analysis was performed to investigate the enriched metabolic pathways associated with ovarian reserve function, and an overexpression analysis of the differentially oxidized lipid metabolites was performed to reveal the biological functional background of FF metabolic disorders in infertile patients with DOR. According to the results of the KEGG Mapper Search, differentially oxidized lipid metabolites were involved in metabolic pathways, including arachidonic acid (AA) metabolism, metabolic pathways, serotonergic synapse, neuroactive ligand-receptor interaction, asthma, phospholipase D signaling pathway, vascular smooth muscle contraction, oxytocin signaling pathway, FC epsilon RI signaling pathway, African trypanosomiasis, bile secretion, and ovarian steroidogenesis. In addition, we input 15 distinct metabolites into the MetaboAnalyst 5.0 database for metabolic pathway enrichment analysis. Only one major AA metabolic pathway was discovered using the Holm $P$-value, false discovery rate (FDR), and Impact value. As a result, when combined with the findings of the KEGG 


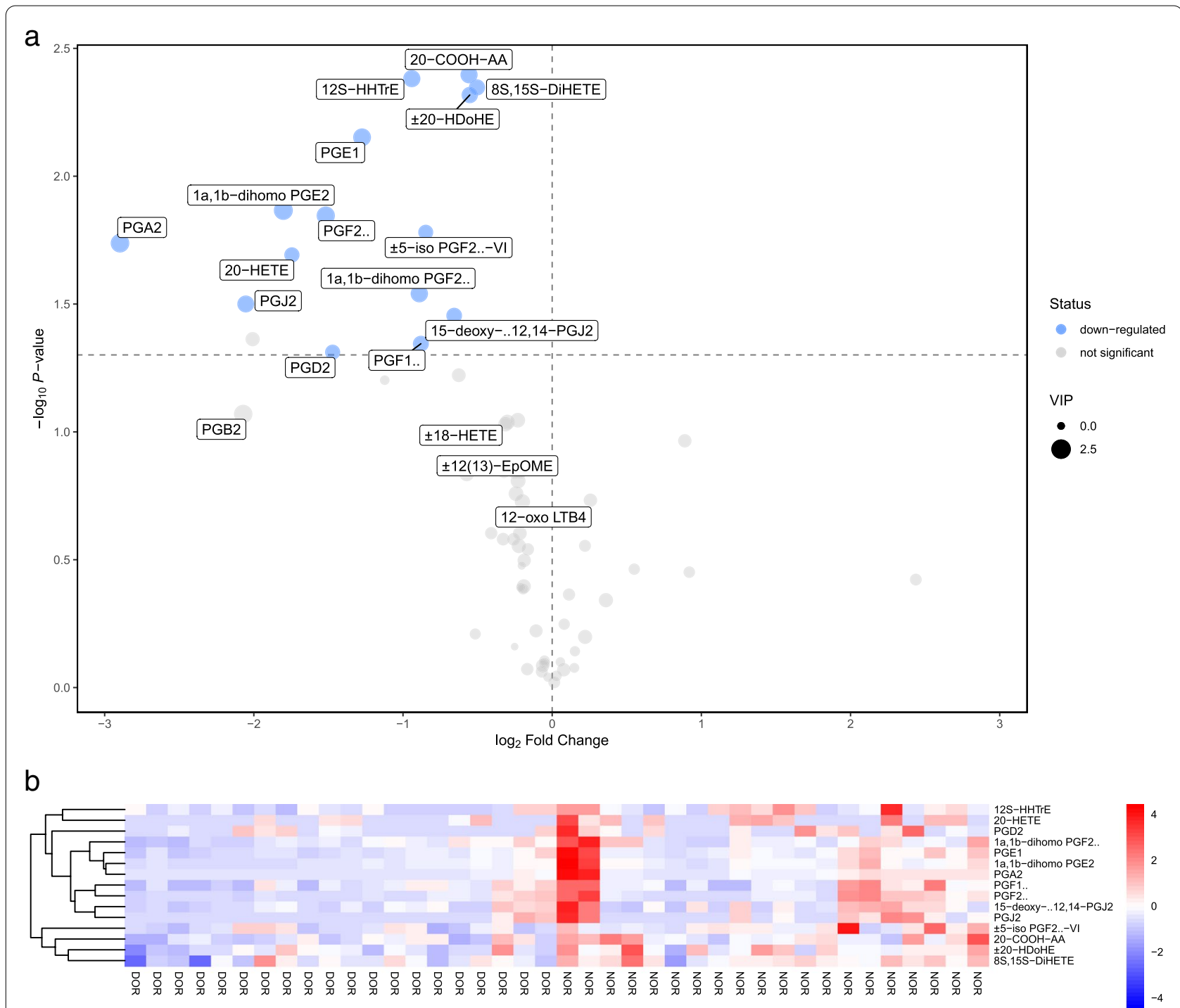

Fig. 2 Identification of the differential metabolomics profiles of FF between DOR and NOR patients based on a volcano plot and hierarchical clustering analysis. a. Volcano plot, down-regulated and up-regulated metabolites in DOR compared to NOR are marked in blue and red, respectively. The $X$-axis represents the log2 fold change of metabolites, while the $Y$-axis represents the fold change of the -log $10 P$ value determined by the Student's t-test. The variable importance in the projection (VIP) value is represented by the dot size. $\mathbf{b}$. Heatmap of the hierarchical clustering analysis. There are fifteen distinct metabolites presented

database analysis, it was hypothesized that the AA metabolic pathway was closely related to DOR (Fig.4).

\section{Predictive value of 15 oxidized lipid metabolites in follicular fluid on ovarian reserve function}

Using ROC curve analysis, the predictive value of 15 oxidized lipid metabolites in ovarian reserve function was determined, and the AUC, 95\% CI, specificity, and sensitivity were obtained. At the same time, the well-known ovarian reserve function markers such as age, FSH, AMH, and AFC, were assessed and used as a reference for lipid metabolite oxidation (Table 3).
The levels of \pm 20 -HDoHE (AUC $=0.782$ ), $12 \mathrm{~S}$ - $\mathrm{HHTrE}$ $(\mathrm{AUC}=0.762), 20-\mathrm{COOH}-\mathrm{AA} \quad(\mathrm{AUC}=0.758), 8 \mathrm{~S}, 15 \mathrm{~S}$ DiHETE $(\mathrm{AUC}=0.800), \mathrm{PGA}_{2}(\mathrm{AUC}=0.850)$, and $\mathrm{PGE}_{1}$ $(\mathrm{AUC}=0.818)$ in FF showed high sensitivity and specificities for the ovarian reserve function, with predictive values comparable to age $(\mathrm{AUC}=0.805)$. Furthermore, we found that the AUC values of FSH, AMH, and AFC were all greater than 0.95 , which was superior to the conventional study to assess their clinical value [20]. The reason for the analysis could be that the patients in this study adhered to FSH, AMH, and AFC standards, demonstrating that the patients included are credible. 

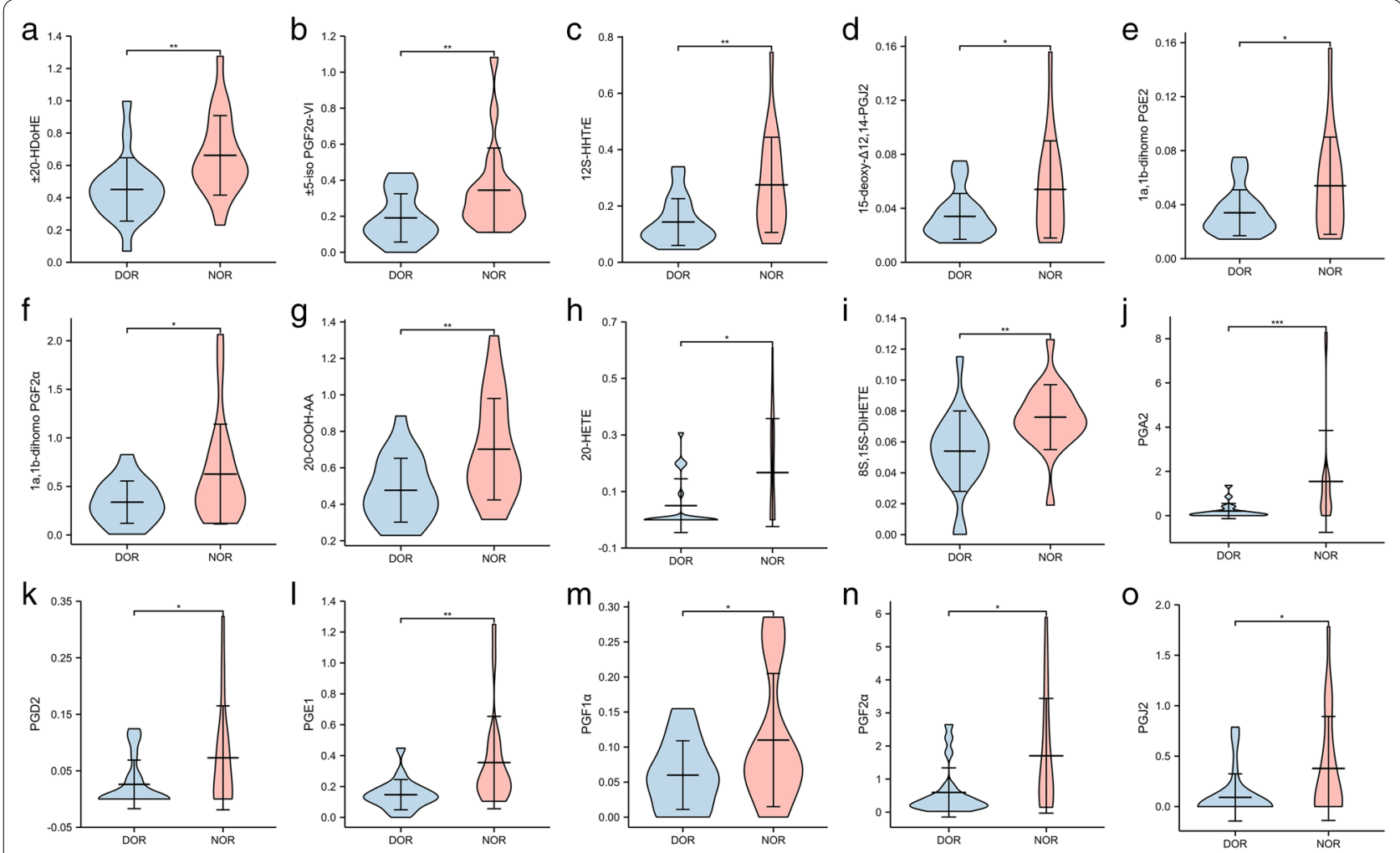

Fig. 3 a-o The violin diagram shows the concentration of 15 different metabolite profiles in the quantitative analysis of DOR and NOR groups. The $y$-axis represents integration data for mass spectral concentration. Each component group is represented by the $x$-axis. ${ }^{*} P<0.05$, ${ }^{* * P}<0.01$, ${ }^{* *} \mathrm{P}<0.001$

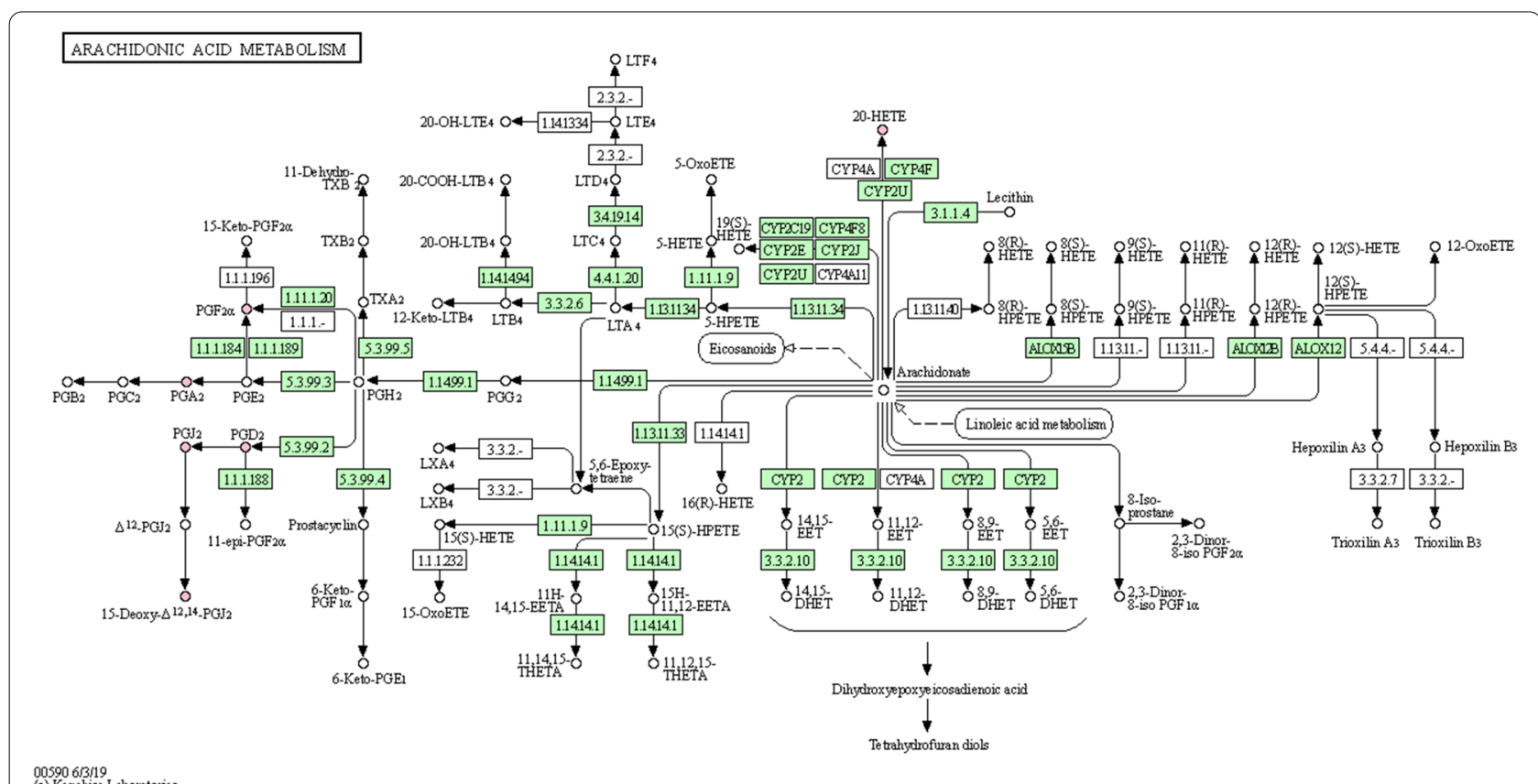

Fig. 4 KEGG's metabolic pathway for arachidonic acid. The pink dots represent differentially expressed metabolites involved in the arachidonic acid metabolic pathway, all of which were reduced in DOR patients. KEGG, Kyoto Encyclopedia of Genes and Genomes; DOR, diminished ovarian reserve 
Table 3 The ROC reports between DOR and NOR group

\begin{tabular}{|c|c|c|c|c|c|c|}
\hline Metabolites & AUC & $95 \% \mathrm{Cl}$ & Cut-off & Sens & Spec & Sens+Spec \\
\hline $\pm 20-\mathrm{HDoHE}$ & 0.782 & $0.632-0.933$ & 0.461 & 0.900 & 0.600 & 1.500 \\
\hline \pm 5 -iso $\mathrm{PGF}_{2 a^{-} \mathrm{Vl}}$ & 0.740 & $0.579-0.901$ & 0.171 & 0.900 & 0.650 & 1.550 \\
\hline 12S-HHTrE & 0.762 & $0.612-0.913$ & 0.138 & 0.800 & 0.650 & 1.450 \\
\hline 15 -deoxy- $\Delta 12,14-P G J_{2}$ & 0.667 & $0.492-0.843$ & 0.045 & 0.600 & 0.850 & 1.450 \\
\hline 1a,1b-dihomo $\mathrm{PGE}_{2}$ & 0.667 & $0.492-0.843$ & 0.045 & 0.600 & 0.850 & 1.450 \\
\hline 1a,1b-dihomo $\mathrm{PGF}_{2 a}$ & 0.680 & $0.512-0.848$ & 0.372 & 0.650 & 0.650 & 1.300 \\
\hline 20-COOH-AA & 0.758 & $0.606-0.909$ & 0.469 & 0.900 & 0.550 & 1.450 \\
\hline 20-HETE & 0.665 & $0.516-0.814$ & 0.229 & 0.450 & 0.950 & 1.450 \\
\hline 8S,15S-DiHETE & 0.800 & $0.655-0.945$ & 0.060 & 0.900 & 0.650 & 1.550 \\
\hline $\mathrm{PGA}_{2}$ & 0.850 & $0.729-0.971$ & 0.232 & 0.800 & 0.800 & 1.600 \\
\hline $\mathrm{PGD}_{2}$ & 0.657 & $0.498-0.817$ & 0.045 & 0.550 & 0.800 & 1.350 \\
\hline $\mathrm{PGE}_{1}$ & 0.818 & $0.687-0.948$ & 0.170 & 0.800 & 0.700 & 1.500 \\
\hline $\mathrm{PGF}_{1 a}$ & 0.644 & $0.470-0.818$ & 0.057 & 0.750 & 0.500 & 1.250 \\
\hline $\mathrm{PGF}_{2 a}$ & 0.735 & $0.576-0.894$ & 0.660 & 0.650 & 0.800 & 1.450 \\
\hline$P G J_{2}$ & 0.675 & $0.535-0.815$ & 0.127 & 0.500 & 0.850 & 1.350 \\
\hline Age & 0.805 & $0.661-0.949$ & 33.50 & 0.950 & 0.650 & 1.600 \\
\hline $\mathrm{FSH}$ & 0.957 & $0.902-1.000$ & 7.545 & 0.900 & 0.900 & 1.800 \\
\hline $\mathrm{AMH}$ & 1.000 & $1.000-1.000$ & 1.820 & 1.000 & 1.000 & 2.000 \\
\hline AFC & 1.000 & $1.000-1.000$ & 7.500 & 1.000 & 1.000 & 2.000 \\
\hline
\end{tabular}

ROC receiver operating characteristic, DOR diminished ovarian reserve, NOR normal ovarian reserve, AUC area under the curve, $95 \% C I 95 \%$ confidence interval, Sens Sensitivity, Spec Specificity, Sens+Spec sensitivity+specificity

\section{Correlation analysis of oxidized lipid metabolite concentrations and related parameters}

Spearman correlation analysis was used to investigate the relationships between different oxidized lipid metabolites in FF and age, FSH, AMH, AFC, retrieved oocytes, MII oocytes, fertilization, high-quality embryos. The concentrations of 20-HDoHE, \pm 5 -iso $\mathrm{PGF}_{2 \alpha}$-VI, 15-deoxy$\Delta 12,14-\mathrm{PGJ}_{2}, 1 \mathrm{a}, 1 \mathrm{~b}$-dihomo $\mathrm{PGE}_{2}, 20-\mathrm{COOH}-\mathrm{AA}, \mathrm{PGA}_{2}$, $\mathrm{PGD}_{2}$, and $\mathrm{PGE}_{1}$ were found to be negatively correlated with FSH. Significant positive correlations were found between 20-HDoHE, \pm 5 -iso $\mathrm{PGF}_{2 \alpha}$-VI, 12S-HHTrE, 1a,1b-dihomo $\mathrm{PGE}_{2}$, 20-COOH-AA, 8S,15S-DiHETE, $\mathrm{PGA}_{2}, \mathrm{PGE}_{1}, \mathrm{PGF}_{2 \alpha}$, and $\mathrm{AMH}$. The concentrations of 20-HDoHE, \pm 5 -iso $\mathrm{PGF}_{2 \alpha}$-VI, 1a,1b-dihomo $\mathrm{PGE}_{2}$, 20-COOH-AA, 8S,15S-DiHETE, PGA, $\mathrm{PGE}_{1}$, and $\mathrm{PGF}_{2 \alpha}$ were positively correlated with AFC. The oocytes retrieval was positively correlated with 20-HDoHE, \pm 5 -iso PGF2 $\alpha$-VI, $12 \mathrm{~S}$-HHTrE, 1a,1b-dihomo $\mathrm{PGE}_{2}$, 20-COOH-AA, 8S,15SDiHETE, PGA,$\quad \mathrm{PGE}_{1}$, and $\mathrm{PGF}_{2 \alpha}$. MII oocytes and fertilization were positively correlated with the concentrations of 20-HDoHE,12SHHTrE,1a,1b-dihomo PGE 2 , 20-COOH-AA, 8S,15SDiHETE, $\mathrm{PGA}_{2}, \mathrm{PGD}_{2}$, and $\mathrm{PGF}_{2 \alpha}$. Only 20-COOH-AA had a positive correlation with high-quality embryos (Table 4).

\section{Discussion}

Oxylipins, also known as lipid mediators, are a class of oxidative metabolites produced by the autooxidation of polyunsaturated fatty acids (AA, linoleic acid, alphalinolenic acid, DHA, EPA, etc.) or by cyclooxygenase (COX), lipoxygenase (LOX), and cytochrome P450 (CYP) enzymes. Oxylipins are signal transduction molecules that participate in nearly all physiological functions of the body and play a critical regulatory role in the organism's life activities, including inflammatory response, immune defense, endocrine regulation, oxidative stress, etc [21]. Besides, it is closely related to the occurrence and development of a variety of diseases, including tumor [22], cardiovascular disease [23], diabetes [24], lung disease [25], and Alzheimer's disease [26]. However, no studies have been conducted to investigate the relationship between oxylipins and the occurrence and development of DOR. Therefore, we performed a UHPLC-MS-MS analysis of the composition of FF oxylipins metabolites in patients with DOR and NOR. Our findings demonstrated changes in oxylipins metabolites in FF of infertile patients with DOR, as well as changes in ovarian reserve function in infertile patients with DOR. We identified 15 oxylipins metabolites that were associated with DOR, including \pm 20 -HDoHE, \pm 5 -iso PGF $_{2 \alpha}$-VI, 12S-HHTrE, 15-deoxy$\triangle 12,14-\mathrm{PGJ}_{2}$, 1a,1b-dihomo PGE 2 , 1a,1b-dihomo PGF $\mathrm{P}_{2 \alpha}$, 20-COOH-AA, 20-HETE, 8S,15S-DiHETE, PGA 2 , PGD ${ }_{2}$, 
Table 4 Correlation analysis between the concentration of oxidized lipid metabolites and related parameters

\begin{tabular}{|c|c|c|c|c|c|c|c|c|c|}
\hline Metabolites(ng/ml) & $\mathrm{R} / P$ & AGE & $\mathrm{FSH}$ & AMH & AFC & OR & MII & FER & HQE \\
\hline \multirow[t]{2}{*}{ 20-HDoHE } & $\mathrm{R}$ & -0.120 & $-0.555^{* *}$ & $0.426^{* *}$ & $0.581^{* *}$ & $0.446^{* *}$ & $0.457^{* *}$ & $0.400^{*}$ & 0.250 \\
\hline & $P$ & 0.461 & 0.000 & 0.006 & 0.000 & 0.004 & 0.003 & 0.010 & 0.120 \\
\hline \multirow[t]{2}{*}{ \pm 5-iso PGF2a-VI } & $\mathrm{R}$ & -0.175 & $-0.455^{* *}$ & $0.361^{*}$ & $0.508^{* *}$ & $0.361^{*}$ & 0.341 & 0.273 & 0.103 \\
\hline & $P$ & 0.286 & 0.004 & 0.024 & 0.001 & 0.024 & 0.052 & 0.093 & 0.531 \\
\hline \multirow[t]{2}{*}{ 12S-HHTrE } & $\mathrm{R}$ & -0.101 & -0.303 & $0.365^{*}$ & 0.252 & $0.347^{*}$ & $0.385^{*}$ & $0.361^{*}$ & 0.002 \\
\hline & P & 0.535 & 0.057 & 0.021 & 0.117 & 0.028 & 0.014 & 0.022 & 0.989 \\
\hline \multirow{2}{*}{15 -deoxy- $\triangle 12,14-P G J_{2}$} & $\mathrm{R}$ & 0.024 & $-0.346^{*}$ & 0.264 & 0.298 & 0.144 & 0.147 & 0.096 & -0.266 \\
\hline & P & 0.882 & 0.029 & 0.099 & 0.062 & 0.376 & 0.365 & 0.556 & 0.097 \\
\hline \multirow[t]{2}{*}{ 1a,1b-dihomo PGE2 } & $\mathrm{R}$ & -0.147 & $-0.347^{*}$ & $0.547^{* *}$ & $0.425^{* *}$ & $0.463^{* *}$ & $0.495^{* *}$ & $0.497^{* *}$ & 0.151 \\
\hline & P & 0.367 & 0.028 & 0.000 & 0.006 & 0.003 & 0.001 & 0.001 & 0.351 \\
\hline \multirow[t]{2}{*}{ 1a,1b-dihomo PGF2a } & $\mathrm{R}$ & 0.052 & -0.148 & 0.259 & 0.296 & 0.223 & 0.276 & 0.287 & 0.056 \\
\hline & $P$ & 0.749 & 0.361 & 0.107 & 0.063 & 0.166 & 0.084 & 0.073 & 0.730 \\
\hline \multirow[t]{2}{*}{ 20-COOH-AA } & $\mathrm{R}$ & -0.022 & $-0.353^{*}$ & $0.390^{*}$ & $0.362^{*}$ & $0.393^{*}$ & $0.414^{* *}$ & $0.425^{* *}$ & $0.339 *$ \\
\hline & P & 0.892 & 0.025 & 0.013 & 0.022 & 0.012 & 0.008 & 0.006 & 0.032 \\
\hline \multirow[t]{2}{*}{ 20-HETE } & $\mathrm{R}$ & 0.191 & -0.386 & 0.327 & 0.447 & 0.510 & 0.436 & 0.412 & -0.220 \\
\hline & $P$ & 0.494 & 0.156 & 0.234 & 0.095 & 0.052 & 0.104 & 0.128 & 0.431 \\
\hline \multirow[t]{2}{*}{ 8S,15S-DiHETE } & $\mathrm{R}$ & -0.023 & -0.241 & $0.509^{* *}$ & $0.564^{* *}$ & $0.452^{* *}$ & $0.377^{*}$ & $0.356^{*}$ & 0.099 \\
\hline & P & 0.889 & 0.145 & 0.001 & 0.000 & 0.004 & 0.020 & 0.028 & 0.553 \\
\hline \multirow[t]{2}{*}{ PGA2 } & $\mathrm{R}$ & -0.149 & $-0.504^{* *}$ & $0.511^{* *}$ & $0.503^{* *}$ & $0.408^{*}$ & $0.424^{*}$ & $0.373^{*}$ & -0.192 \\
\hline & P & 0.408 & 0.003 & 0.002 & 0.003 & 0.019 & 0.014 & 0.033 & 0.284 \\
\hline \multirow[t]{2}{*}{ PGD2 } & $\mathrm{R}$ & -0.187 & $-0.551^{*}$ & 0.009 & 0.385 & 0.374 & $0.482^{*}$ & $0.463^{*}$ & -0.168 \\
\hline & P & 0.442 & 0.015 & 0.972 & 0.104 & 0.115 & 0.037 & 0.046 & 0.492 \\
\hline \multirow[t]{2}{*}{ PGE1 } & $\mathrm{R}$ & -0.178 & $-0.481^{* *}$ & $0.559^{* *}$ & $0.512^{* *}$ & $0.442^{* *}$ & $0.437^{* *}$ & $0.405^{*}$ & -0.058 \\
\hline & $P$ & 0.278 & 0.002 & 0.000 & 0.001 & 0.005 & 0.005 & 0.011 & 0.726 \\
\hline \multirow[t]{2}{*}{ PGF1a } & $\mathrm{R}$ & -0.085 & -0.323 & 0.278 & 0.297 & 0.128 & 0.131 & 0.148 & -0.287 \\
\hline & P & 0.644 & 0.071 & 0.124 & 0.099 & 0.485 & 0.475 & 0.418 & 0.111 \\
\hline \multirow[t]{2}{*}{ PGF2a } & $\mathrm{R}$ & -0.162 & -0.288 & $0.383^{*}$ & $0.357^{*}$ & $0.344^{*}$ & $0.414^{* *}$ & $0.404^{*}$ & -0.074 \\
\hline & $P$ & 0.319 & 0.072 & 0.015 & 0.024 & 0.030 & 0.008 & 0.010 & 0.649 \\
\hline \multirow[t]{2}{*}{$\mathrm{PGJ}_{2}$} & $\mathrm{R}$ & -0.100 & -0.330 & 0.071 & 0.339 & 0.014 & 0.196 & 0.025 & -0.422 \\
\hline & P & 0.745 & 0.271 & 0.817 & 0.257 & 0.964 & 0.520 & 0.936 & 0.151 \\
\hline
\end{tabular}

$P, P$-value, ${ }^{* *} P<0.01,{ }^{*} P<0.05 ; \mathrm{AGE}$, age; FSH, follicle stimulating hormone; AMH, anti-Mullerian hormone; AFC, antral follicle count; OR, number of oocytes retrieved; MII number of MII oocytes; FER, number of fertilizations, $\mathrm{HQE}$, number of high-quality embryos

$\mathrm{PGE}_{1}, \mathrm{PGF}_{1 \alpha}, \mathrm{PGF}_{2 \alpha}$, and PGJ ${ }_{2}$. The levels of the 15 differentially oxidized lipid metabolites in the FF of DOR patients were all lower than in the NOR group, and the differences were statistically significant. Based on the KEGG and Metaboanalyst database, the results of pathway enrichment analysis showed that the differential oxylipins metabolites were closely related to the AA metabolic pathway. These distinct oxylipins metabolites and the AA metabolic pathway may play a non-negligible role in DOR infertility. Dysfunctions in oxylipins metabolism in FF of DOR patients related to follicular development may provide potential diagnostic and therapeutic targets for promoting oocyte maturation.

Polyunsaturated fatty acids (PUFAs) are long-chain fatty acids with two or more double bonds, the n-3 and n-6 series fatty acids having biological significance. Imbalanced dietary intake of n-6 to n-3 PUFAs has been associated with cardiovascular and cerebrovascular diseases, cancer, inflammation, and autoimmune diseases [27]. Wathes et al. [28] demonstrated that dietary n-6 and $\mathrm{n}$-3PUFAs can alter reproductive processes by acting as precursors for prostaglandin synthesis and by regulating the expression patterns of many key enzymes involved in prostaglandin and steroid metabolism. AA is an $n-6$ PUFA essential fatty acid that is cleaved from phospholipids by phospholipase A2 (PLA2), phosphatidyl inositol specific phospholipase $C$, and the fatty acylglycerol lipase. AA is then metabolized to eicosanoids via COX, LOX, or P450-dependent cyclooxygenase pathways [29]. A previous study demonstrated that high concentrations of AA derivatives in human FF at the time of oocyte retrieval significantly decreased oocyte's ability to form pronuclei after ICSI [30]. As cell signaling intermediates, AA and its derivatives regulate cAMP activation, $\mathrm{Ca}^{2+}$ 
influx, $\mathrm{Ca}^{2+} / \mathrm{CaM}, \mathrm{PKC}, \mathrm{MAPK}$, and PI3K/Akt, resulting in regulation of cellar growth, proliferation, and differentiation [31,32]. Another study reported that AA is one of the most abundant polyunsaturated fatty acids [33]. The concentration of AA in FF in bovine ovaries accounts for about $2.5 \%$ of total fatty acids, whereas the concentration in plasma is $1.2 \%$ [34]. It has been reported that follicular AA regulates cumulus granulosa cells in human [35] and non-human mammals [33]. Zhang et al. [36] demonstrated that AA regulates the survival, gene expression, lipid formation, steroid production, and intracellular signaling pathways in bovine granulosa cells. This supports the hypothesis that AA in FF can directly influence the function of granulosa cells, thereby modulating follicular development and ovulation. Khajeh et al. [37] investigated the biological effects of AA in human cumulus granulosa cells (CGCs) after exposure to acetylsalicylic acid (ASA). They found that ASA treatment reduced $E_{2}$ production, Cyp19a1 expression, GSH-Px activity, and estradiol receptor expression in CGCs. Importantly, the addition of AA abolished the ASA-induced reduction in $\mathrm{E}_{2}$ levels and expression of Cyp19a1, increased the antioxidant capacity of CGCs exposed to ASA by increasing GSH-Px activity, synthesis, and secretion of $\mathrm{PGE}_{2}$, and increasing the expression of the estrogen receptors. $\mathrm{Li}$ et al. [38] found that the levels of AA and its metabolites were elevated in FF of patients with PCOS, and insulin increased the generation of AA metabolites by COX-2. This is likely to be a novel molecular pathophysiological mechanism of PCOS. In the present study, we found that the level of AA metabolites in FF of patients with DOR was lower compared to its level in the NOR control group, and these decreased AA metabolites were generated via COX-2 and CYP pathway, but not LOX pathway. CYP enzymes mediate AA transformation into 20-hydroxyeicosatetraenoic acid (20-HETE) [39]. A study revealed a higher serum concentration of 20-HETE in obese women as compared to that in women with normal weight [40]. Elsewhere, 20-HETE concentration in urine and plasma of patients with metabolic syndrome was found to be higher as compared to that of control [41]. There is more evidence of a significant correlation between the level of 20-HETE in blood with BMI, whereby higher BMI denote higher levels of 20-HETE [42]. 20-HETE significantly impacts obesity associated with a high-fat diet, impaired insulin signaling, and insulin resistance (IR) [43]. Li et al. [38] found higher levels of 20-HETE in the FF of non-obese PCOS patients that underwent IVF as compared to that in the non-PCOS group, but the difference was not significant [38]. We, herein, reported lower levels of 20-HETE in FF of DOR patients as compared to that of subjects in the NOR control group. However, the correlation and mechanism between 20-HETE and DOR remain elusive.

AA is a well-known precursor of prostaglandins (PGs), which are synthesized PGs by prostaglandin-endoperoxide synthase S1 (PTGS1) or PTGS-2 previously identified as cyclooxygenase enzymes COX1 and COX2 [44, 45]. PTGS-2(COX2) is the critical prostaglandin-endoperoxide synthase in the ovary. Mounting evidence indicates that PTGS-2(COX2) deficient female mice exhibit multiple failures in female reproductive processes, including ovulation, fertilization, implantation, and decidualization $[46,47]$. Prostaglandins, especially $\mathrm{PGE}_{2}$ and $\mathrm{PGF}_{2 \alpha}$, have previously been shown to regulate oocyte maturation, ovulation, and cumulus expansion [48, 49]. Additional evidence indicates that $\mathrm{PGE}_{2}$ plays a crucial role in protecting oocytes against oxidative stress, therefore, holds promise as a novel autocrine/paracrine player in the mechanisms that potentially drive successful oocyte maturation and oocyte survival in cow [50]. Moreover, $\mathrm{PGF}_{2 \alpha}$ is synthesized in response to LH surge, however, its underlying role in the ovulatory process remains to be determined [51]. Some researchers suggest that both $\mathrm{PGF}_{2 \alpha}$ and $\mathrm{PGE}_{2}$ are important in the ovulatory process [52, 53]. Pereira de Moraes et al. [54], however, concluded that $\mathrm{PGF}_{2 \alpha}$ alone is unable to induce ovulation in cattle. Sharma et al. [55] investigated $\mathrm{PGE}_{2}$ treated follicles and revealed a reduction in the attributes of apoptosis in granulosa cells, whereas $\mathrm{PGF}_{2 \alpha}$ saw an increase in apoptotic characteristics. Furthermore, Kemiläinen et al. [56] found a broad expression of HSD17B12 enzyme in both human and mouse ovaries, demonstrating a role for HSD17B12 in the synthesis of $\mathrm{PGD}_{2}, \mathrm{PGE}_{2}, \mathrm{PGF}_{2 \alpha}$, and TXB2, which are well-known prostaglandins for their regulatory role in ovarian function. Another product of the AA cyclooxygenase pathway is 15 -deoxy- $\Delta 12,14$ $\mathrm{PGJ}_{2}$, a specific endogenous ligand for a peroxisome proliferator-activated receptor $\gamma$ (PPAR $\gamma$ ) in vivo [57]. PPAR $\gamma$ was detected in primary and secondary follicles, but was expressed highly in the large follicles, and is thought to play a role in regulating the expression of genes involved with growth, development, and/or differentiation of the follicle. Treatment of granulosa cells with 15-deoxy- $\Delta 12,14-\mathrm{PGJ}_{2}$ stimulated basal progesterone secretion but elicited no significant effect on FSH-stimulated steroid production, suggesting a potential role for PPAR $\gamma$ in regulating genes related to follicular differentiation [58]. Also, the PPARy decline in response to $\mathrm{LH}$ is important for ovulation and/or luteinization [58]. Significantly higher levels of AA metabolites (such as PGE ${ }_{2}$, $\mathrm{PGD}_{2}, \mathrm{PGJ}_{2}, 15-$ deoxy- $\Delta 12,14-\mathrm{PGJ}_{2}$, and $\mathrm{PGF}_{2 \alpha}$ ) produced via the COX pathway were found in FF of PCOS patients as compared to those of the non-PCOS group [38]. In the present study, the levels of AA metabolites 
(such as $\mathrm{PGE}_{2}, \mathrm{PGD}_{2}, \mathrm{PGI}_{2}, 15$-deoxy- $\Delta 12,14-\mathrm{PGI}_{2}$, and $\mathrm{PGF}_{2 \alpha}$ ) produced via the COX pathway in FF of DOR patients were lower than those in the NOR control group. However, the specific mechanism of DOR and the level of metabolites produced by the arachidonic acid COX enzyme metabolic pathway remain elusive.

Our analysis revealed 15 types of differentially oxidized lipid metabolites in FF, possessing certain predictive value for ovarian reserve function. In particular, the levels of \pm 20 -HDoHE, 12S-HHTrE, 20-COOH-AA, 8S,15SDiHETE, $\mathrm{PGA}_{2}$, and $\mathrm{PGE}_{1}$ showed high sensitivities and specificities. Through Spearman correlation analysis, we also explored the correlations between the concentration of differentially oxidized lipid metabolites in FF and age, FSH, AMH, AFC, oocytes retrieved, MII oocytes, fertilization, high-quality embryos. Results demonstrated a negative correlation between the concentrations of eight lipid oxidative metabolites in FF with FSH but a positive correlation with AFC. Nine types of oxidized lipid metabolites were positively correlated with $\mathrm{AMH}$, the number of oocytes retrieved, MII oocytes, and fertilization. Notably, one metabolite was positively correlated with the number of high-quality embryos. The changes in the content of oxidized lipid metabolites in FF of DOR patients suggest their potential role in oocyte development, maturation, fertilization, and early embryo development. However, it remains elusive as to how the above lipid-oxidizing metabolites play a role in FF and which metabolic regulation channels influence ovarian reserve function and oocyte quality, which warrants further exploration.

Intriguingly, our analysis showed that the differential oxidized lipid metabolites in FF of DOR patients were lower than those of NOR patients, which contradicts our original hypothesis of the search for markers of oxidized lipid metabolism in FF of DOR patients. The possible reasons may be that: First, our reproductive center routinely employed a double-cavity needle to wash oocytes, allowing DOR patients to obtain maximum levels of oocytes, which is not the case for NOR patients. As such, we insinuate the potential influence of needle washing liquid on oxidized lipid metabolites in FF. Second, the structure of some oxidized lipid metabolites in FF may be unstable; however, we made our best attempt to ensure that the processes of collecting, transporting, centrifuging, and shipping FF for detection have no underlying impact. It is suggested that the above process may still impact oxidized lipid metabolites in FF. Furthermore, because the limited amount of oxidized lipid metabolites that can be detected by oxidized lipid metabolomics at present, we speculate that the different oxidative lipid metabolites in FF of DOR and NOR patients are way much limited, to an extent that the up-regulated metabolites may not be detected. Finally, it is possible that we analyzed a small number of samples and the individual differences of clinical samples were large. Although patients were screened with strict adherence to the inclusion criteria, the influence of individual differences cannot be ignored. Therefore, the difference between DOR and NOR patients in the up-regulated oxidized lipid metabolites in FF is not significant. Another finding of this study is that the down-regulated oxidized lipid metabolites in FF of DOR patients are primarily linked to AA metabolism. Mounting evidence indicates that AA potentially induces and inhibits oxidative stress, for instance, it can increase calcium influx by activating the calcium channel on the cell membrane $[59,60]$, which subsequently activates $\mathrm{NADPH}$ oxidase, increasing ROS levels and induces oxidative stress damage [61]. AA can also activate the mRNA and protein levels of PPAR, ameliorate the activities of SOD, CAT, and GSH-Px, decrease the production of mitochondrial ROS to arrest oxidative stress injury [62]. These data strongly suggest a dominant role for AA and its metabolites in anti-oxidative stress in FF of DOR patients, which will be explored in our future study.

\section{Conclusions}

Through UHPLC-MS/MS, oxylipins metabolites changes in FF of the DOR and NOR patients were investigated. Metabonomic analysis of FF showed 15 differentially expressed oxylipins metabolites, associated with ovarian reserve function. The differentially oxidized lipid metabolites were lower in FF of DOR patients as compared to those of the NOR group. The pathway enrichment analysis demonstrated that the differentially oxidized lipid metabolites are mainly concentrated in the AA metabolic pathway, while AA is involved in the regulation of oocyte development and maturation, and its complex changes are closely related to follicular development. The differential oxylipins metabolites and AA metabolic pathway may play a significant role in DOR infertility. Dysfunctions in the metabolism of oxylipins related to follicular development in FF of DOR patients may provide potential detection avenues and therapeutic targets for promoting oocyte maturation. The findings may also provide a scientific basis for understanding the microenvironment of oocyte development and improving oocyte quality in patients with DOR.

\section{Abbreviations}

DOR: Diminished ovarian reserve; POF: Premature ovarian failure; POR: Poor ovarian responder; NOR: Normal ovarian reserve; IVF: In vitro fertilization;

ICSI-ET: intracytoplasmic sperm injection-embryo transfer; OS: Oxidative stress; ROS: Reactive oxygen species; MDA: Malondialdehyde; FF: Follicular fluid; AGE: Advanced glycation end-products; AMH: Anti-Mullerian hormone; FSH: Follicle stimulating hormone; LH: Luteinizing hormone; bAFC: Basic antral follicle count; $E_{2}$ : Estrogen; P: Progesterone; SWATH: Sequential window acquisition 
of all theoretical fragment-ion spectra; MRM: Multiple reaction monitor; PCA: Principal component analysis; OPLS-DA: Orthogonal projections to latent structure discriminant analysis; QC: Quality control; VIP: Variable importance in the projection; KEGG: Kyoto Encyclopedia of Genes and Genomes; BMI: Body mass index; HMG: Human menopausal gonadotropin; HCG: Human chorionic gonadotropin; SOD: Superoxide dismutase; CAT: Catalase; GSH-PX: Glutathione peroxidase; AA: Arachidonic acid; FDR: False discovery rate; COX: Cyclooxygenase; LOX: Lipoxygenase; CYP: Cytochrome P450; PUFAs: Polyunsaturated fatty acids; PLA2: Phospholipase A2; CGCs: Cumulus granulosa cells; ASA: Acetylsalicylic acid; 20-HETE: 20-hydroxyeicosatetraenoic acid; IR: Insulin resistance; PGs: Prostaglandins; PTGS1: Prostaglandin-endoperoxide synthase S1; PTGS2: Prostaglandin-endoperoxide synthase S2; PPARY: Peroxisome proliferatoractivated receptor $\gamma$; ROC: Receiver operating characteristic; AUC: Area under the curve.

\section{Acknowledgements}

We are thankful to all the participants of the study. We would like to thank Shanghai Biotree Biotechnology for the UHPLC-MS/MS and data analyses.

\section{Authors' contributions}

LCC performed the literature search, data analysis and interpretation, and wrote the manuscript. $Z X \mathrm{~L}$ and $\mathrm{HH}$ constructed the tables and figures. LCC and ZXX performed the sample collection and data analysis. ZQH, QC and FYL contributed to the design of the research study and provided critical review of the manuscript. All authors read and approved the final manuscript.

\section{Funding}

This work was supported by the Key Project of the National Key Research and Development Program of "Modernization Research of Traditional Chinese Medicine" -Systematic Study on the Criteria for Differentiation of "Kidney-Yang Deficiency Syndrome" (No. SQ2018YFC170061)

\section{Availability of data and materials}

The datasets used and analyzed during the current study are available from the corresponding author on reasonable request.

\section{Declarations}

\section{Ethics approval and consent to participate}

This study was approved by the Research Ethics Committees of Shuguang hospital affiliated to Shanghai university of traditional Chinese medicine (No.2020-833-40-01). All participants signed an informed consent form prior to the study.

\section{Consent for publication}

Not applicable.

\section{Competing interests}

The authors declare that they have no conflict of interest.

\section{Author details}

'Shanghai University of Traditional Chinese Medicine, Shanghai 201203, China. ${ }^{2}$ Department of Gynecology, Shuguang Hospital affiliated to Shanghai University of Traditional Chinese Medicine, 201203 Shanghai, China. ${ }^{3}$ Shanghai First Maternity and Infant Hospital, Tongji University School of Medicine, Shanghai 201204, China.

Received: 23 June 2021 Accepted: 2 September 2021

Published online: 14 September 2021

\section{References}

1. Lu Q, Shen H, Li Y, Zhang C, Wang C, Chen X, et al. Low testosterone levels in women with diminished ovarian reserve impair embryo implantation rate: a retrospective case-control study. J Assist Reprod Genet. 2014;31(4):485-91.

2. Mutlu MF, Erdem A. Evaluation of ovarian reserve in infertile patients. J Turk Ger Gynecol Assoc. 2012;13(3):196-203.
3. European Society for Human Reproduction and Embryology (ESHRE) Guideline Group on POI, Webber L, Davies M, Anderson R, Bartlett J, Braat $D$, et al. ESHRE Guideline: management of women with premature ovarian insufficiency. Hum Reprod. 2016;31(5):926-37.

4. Bishop LA, Richter KS, Patounakis G, Andriani L, Moon K, Devine K. Diminished ovarian reserve as measured by means of baseline folliclestimulating hormone and antral follicle count is not associated with pregnancy loss in younger in vitro fertilization patients. Fertil Steril. 2017;108(6):980-7.

5. Nita M, Grzybowski A. The Role of the Reactive Oxygen Species and Oxidative Stress in the Pathomechanism of the Age-Related Ocular Diseases and Other Pathologies of the Anterior and Posterior Eye Segments in Adults. Oxid Med Cell Longev. 2016;2016:3164734.

6. Wohlgemuth SE, Calvani R, Marzetti E. The interplay between autophagy and mitochondrial dysfunction in oxidative stress-induced cardiac aging and pathology. J Mol Cell Cardiol. 2014;71:62-70.

7. Yang Y, Sharma R, Sharma A, Awasthi S, Awasthi YC. Lipid peroxidation and cell cycle signaling: 4-hydroxynonenal, a key molecule in stress mediated signaling. Acta Biochim Pol. 2003;50(2):319-36.

8. Luderer U. Ovarian toxicity from reactive oxygen species. Vitam Horm. 2014;94:99-127.

9. Shamilova NN, Marchenko LA, Dolgushina NV, Zaletaev DV, Sukhikh GT. The role of genetic and autoimmune factors in premature ovarian failure. J Assist Reprod Genet. 2013;30(5):617-22.

10. Razi Y, Eftekhar M, Fesahat F, Dehghani Firouzabadi R, Razi N, Sabour M, et al. Concentrations of homocysteine in follicular fluid and embryo quality and oocyte maturity in infertile women: a prospective cohort. J Obstet Gynaecol. 2020:4:1-6.

11. Agarwal A, Aponte-Mellado A, Premkumar BJ, Shaman A, Gupta S. The effects of oxidative stress on female reproduction: a review. Reprod Biol Endocrinol. 2012;10:49.

12. Jia ZZ, Zhang JW, Zhou D, Xu DQ, Feng XZ. Deltamethrin exposure induces oxidative stress and affects meiotic maturation in mouse oocyte. Chemosphere. 2019;223:704-13.

13. Lord T, Aitken RJ. Oxidative stress and ageing of the post-ovulatory oocyte. Reproduction. 2013;146(6):R217-27.

14. Tian H, Liu K, Zhang Y, Qi Q, Wang C, Guan H, et al. Adult follicular fluid supplementation during in vitro maturation improves the developmental competence of prepubertal lamb oocytes. Theriogenology. 2019;130:157-62.

15. Sreekumar A, Poisson LM, Rajendiran TM, Khan AP, Cao Q, Yu J, et al. Metabolomic profiles delineate potential role for sarcosine in prostate cancer progression. Nature. 2009;457(7231):910-4.

16. Merhi Z, Irani M, Doswell AD, Ambroggio J. Follicular fluid soluble receptor for advanced glycation end-products (SRAGE): a potential indicator of ovarian reserve. J Clin Endocrinol Metab. 2014;99(2):E226-33.

17. Pacella L, Zander-Fox DL, Armstrong DT, Lane M. Women with reduced ovarian reserve or advanced maternal age have an altered follicular environment. Fertil Steril. 2012;98(4):986-94 e1-2.

18. Tal R, Seifer DB. Ovarian reserve testing: a user's guide. Am J Obstet Gynecol. 2017;217(2):129-40.

19. Zhu X, Ye J, Fu Y. Late Follicular Phase Ovarian Stimulation Without Exogenous Pituitary Modulators. Front Endocrinol (Lausanne). 2020;11:487.

20. Zhou SJ, Zhao MJ, Li C, Su X. The comparison of evaluative effectiveness between antral follicle count/age ratio and ovarian response prediction index for the ovarian reserve and response functions in infertile women. Medicine (Baltimore). 2020:99(36):e21979.

21. Gabbs M, Leng S, Devassy JG, Monirujjaman M, Aukema HM. Advances in Our Understanding of Oxylipins Derived from Dietary PUFAs. Adv Nutr. 2015;6(5):513-40.

22. Edin ML, Duval C, Zhang G, Zeldin DC. Role of linoleic acid-derived oxylipins in cancer. Cancer Metastasis Rev. 2020;39(3):581-2.

23. Nayeem MA. Role of oxylipins in cardiovascular diseases. Acta Pharmacol Sin. 2018;39(7):1142-54

24. Tans R, Bande R, van Rooij A, Molloy BJ, Stienstra R, Tack CJ, et al. Evaluation of cyclooxygenase oxylipins as potential biomarker for obesity-associated adipose tissue inflammation and type 2 diabetes using targeted multiple reaction monitoring mass spectrometry. Prostaglandins Leukot Essent Fatty Acids. 2020;160:102157. 
25. Lundström SL, Balgoma D, Wheelock ÅM, Haeggström JZ, Dahlén SE, Wheelock CE. Lipid mediator profiling in pulmonary disease. Curr Pharm Biotechnol. 2011;12(7):1026-52.

26. Devassy JG, Leng S, Gabbs M, Monirujjaman M, Aukema HM. Omega-3 Polyunsaturated Fatty Acids and Oxylipins in Neuroinflammation and Management of Alzheimer Disease. Adv Nutr. 2016;7(5):905-16.

27. Xu H, Dong X, Ai Q, Mai K, Xu W, Zhang Y, et al. Regulation of tissue LCPUFA contents, $\triangle 6$ fatty acyl desaturase (FADS2) gene expression and the methylation of the putative FADS2 gene promoter by different dietary fatty acid profiles in Japanese seabass (Lateolabrax japonicus). PLoS One. 2014;9(1):e87726.

28. Wathes DC, Abayasekara DR, Aitken RJ. Polyunsaturated fatty acids in male and female reproduction. Biol Reprod. 2007;77(2):190-201.

29. Hanna VS, Hafez EAA. Synopsis of arachidonic acid metabolism: A review. J Adv Res. 2018;11:23-32.

30. Ciepiela P, Bączkowski T, Drozd A, Kazienko A, Stachowska E, Kurzawa R. Arachidonic and linoleic acid derivatives impact oocyte ICSI fertilization-a prospective analysis of follicular fluid and a matched oocyte in a 'one follicle--one retrieved oocyte--one resulting embryo' investigational setting. PLoS One. 2015;10(3):e0119087.

31. Cheng Z, Sheldrick EL, Marshall E, Wathes DC, Abayasekara DR, Flint AP. Control of cyclic AMP concentration in bovine endometrial stromal cells by arachidonic acid. Reproduction. 2007;133(5):1017-26.

32. Zuccolo E, Dragoni S, Poletto V, Catarsi P, Guido D, Rappa A, et al. Arachidonic acid-evoked $\mathrm{Ca}^{2+}$ signals promote nitric oxide release and proliferation in human endothelial colony forming cells. Vascul Pharmacol. 2016;87:159-71.

33. Lapa M, Marques CC, Alves SP, Vasques MI, Baptista MC, Carvalhais I, et al. Effect of trans-10 cis-12 conjugated linoleic acid on bovine oocyte competence and fatty acid composition. Reprod Domest Anim. 2011:46(5):904-10.

34. Renaville B, Bacciu N, Comin A, Motta M, Poli I, Vanini G, et al. Plasma and follicular fluid fatty acid profiles in dairy cows. Reprod Domest Anim. 2010;45(1):118-21.

35. Moran LJ, Tsagareli V, Noakes M, Norman R. Altered Preconception Fatty Acid Intake Is Associated with Improved Pregnancy Rates in Overweight and Obese Women Undertaking in Vitro Fertilisation. Nutrients. 2016;8(1):10.

36. Zhang N, Wang L, Luo G, Tang X, Ma L, Zheng Y, et al. Arachidonic Acid Regulation of Intracellular Signaling Pathways and Target Gene Expression in Bovine Ovarian Granulosa Cells. Animals (Basel). 2019;9(6):374.

37. Khajeh M, Nouri M, Ghasemzadeh A, Mehdizadeh A, Shanehbandi D, Yousefi $\mathrm{S}$, et al. Arachidonic acid alleviates the detrimental effects of acetylsalicylic acid on human granulosa cells performance in vitro. Mol Reprod Dev. 2020;87(5):607-19.

38. Li S, Qi J, Tao Y, Zhu Q, Huang R, Liao Y, et al. Elevated Levels of Arachidonic Acid Metabolites in Follicular Fluid of PCOS Patients. Reproduction. 2019; REP-19-0136.R2.

39. Kroetz DL, Xu F. Regulation and inhibition of arachidonic acid omegahydroxylases and 20-HETE formation. Annu Rev Pharmacol Toxicol. 2005:45:413-38.

40. Peterson SJ, Vanella L, Gotlinger K, Jiang H, Singh SP, Sodhi K, et al. Oxidized $\mathrm{HDL}$ is a potent inducer of adipogenesis and causes activation of the Ang-II and 20-HETE systems in human obese females. Prostaglandins Other Lipid Mediat. 2016;123:68-77.

41. Tsai IJ, Croft KD, Mori TA, Falck JR, Beilin LJ, Puddey IB, et al. 20-HETE and F2-isoprostanes in the metabolic syndrome: the effect of weight reduction. Free Radic Biol Med. 2009;46(2):263-70.

42. Issan Y, Hochhauser E, Guo A, Gotlinger KH, Kornowski R, Leshem-Lev D, et al. Elevated level of pro-inflammatory eicosanoids and EPC dysfunction in diabetic patients with cardiac ischemia. Prostaglandins Other Lipid Mediat. 2013;100-101:15-21

43. Gilani A, Pandey V, Garcia V, Agostinucci K, Singh SP, Schragenheim J, et al. High-fat diet-induced obesity and insulin resistance in CYP4a $14^{-/-}$ mice is mediated by 20-HETE. Am J Physiol Regul Integr Comp Physiol. 2018;315(5):R934-44.
44. Kudo I, Murakami M. Prostaglandin E synthase, a terminal enzyme for prostaglandin E2 biosynthesis. J Biochem Mol Biol. 2005;38(6):633-8.

45. Asselin E, Lacroix D, Fortier MA. IFN-tau increases PGE2 production and COX-2 gene expression in the bovine endometrium in vitro. Mol Cell Endocrinol. 1997;132(1-2):117-26.

46. Hester KE, Harper MJ, Duffy DM. Oral administration of the cyclooxygenase-2 (COX-2) inhibitor meloxicam blocks ovulation in non-human primates when administered to simulate emergency contraception. Hum Reprod. 2010;25(2):360-7.

47. Lim H, Paria BC, Das SK, Dinchuk JE, Langenbach R, Trzaskos JM, et al. Multiple female reproductive failures in cyclooxygenase 2-deficient mice. Cell. 1997;91(2):197-208.

48. Takahashi T, Morrow JD, Wang H, Dey SK. Cyclooxygenase-2-derived prostaglandin $\mathrm{E}(2)$ directs oocyte maturation by differentially influencing multiple signaling pathways. J Biol Chem. 2006;281 (48):37117-29.

49. Marei WF, Abayasekara DR, Wathes DC, Fouladi-Nashta AA. Role of PTGS2-generated PGE2 during gonadotrophin-induced bovine oocyte maturation and cumulus cell expansion. Reprod Biomed Online. 2014;28(3):388-400

50. Boruszewska D, Kowalczyk-Zieba I, Suwik K, Staszkiewicz-Chodor J, Jaworska J, Lukaszuk K, et al. Prostaglandin $\mathrm{E}_{2}$ affects in vitro maturation of bovine oocytes. Reprod Biol Endocrinol. 2020;18(1):40.

51. Duffy DM, Ko C, Jo M, Brannstrom M, Curry TE. Ovulation: Parallels With Inflammatory Processes. Endocr Rev. 2019;40(2):369-416.

52. Algire JE, Srikandakumar A, Guilbault LA, Downey BR. Preovulatory changes in follicular prostaglandins and their role in ovulation in cattle. Can J Vet Res. 1992;56(1):67-9.

53. Berisha B, Rodler D, Schams D, Sinowatz F, Pfaffl MW. Prostaglandins in Superovulation Induced Bovine Follicles During the Preovulatory Period and Early Corpus Luteum. Front Endocrinol (Lausanne). 2019;10:467.

54. Pereira de Moraes F, Amaral D'Avila C, Caetano de Oliveira F, Ávila de Castro N, Diniz Vieira A, Schneider A, et al. Prostaglandin F2a regulation and function during ovulation and luteinization in cows. Theriogenology. 2021;171:30-7.

55. Sharma AK, Sharma RK. Effect of prostaglandins $E_{2}$ and $F_{2 a}$ on granulosa cell apoptosis in goat ovarian follicles. Iran JVet Res. 2020;21(2):97-102.

56. Kemiläinen $\mathrm{H}$, Adam M, Mäki-Jouppila J, Damdimopoulou P, Damdimopoulos AE, Kere J, et al. The Hydroxysteroid (17ß) Dehydrogenase Family Gene HSD17B12 Is Involved in the Prostaglandin Synthesis Pathway, the Ovarian Function, and Regulation of Fertility. Endocrinology. 2016;157(10):3719-30.

57. Forman BM, Tontonoz P, Chen J, Brun RP, Spiegelman BM, Evans RM. 15-Deoxy-delta 12, 14-prostaglandin J2 is a ligand for the adipocyte determination factor PPAR gamma. Cell. 1995;83(5):803-12.

58. Komar CM, Curry TE Jr. Inverse relationship between the expression of messenger ribonucleic acid for peroxisome proliferator-activated receptor gamma and P450 side chain cleavage in the rat ovary. Biol Reprod. 2003;69(2):549-55.

59. Fiorio Pla A, Genova T, Pupo E, Tomatis C, Genazzani A, Zaninetti R, et al. Multiple roles of protein kinase a in arachidonic acid-mediated Ca2+ entry and tumor-derived human endothelial cell migration. Mol Cancer Res. 2010;8(11):1466-76.

60. Thompson J, Mignen O, Shuttleworth TJ. The N-terminal domain of Orai3 determines selectivity for activation of the store-independent ARC channel by arachidonic acid. Channels (Austin). 2010;4(5):398-410.

61. Tseng CL, Wei JW. Homologous desensitization of histamine-mediated signal transduction system in C6 glioma cells. Chin J Physiol. 2013;56(2):90-100.

62. Wang ZJ, Liang CL, Li GM, Yu CY, Yin M. Neuroprotective effects of arachidonic acid against oxidative stress on rat hippocampal slices. Chem Biol Interact. 2006;163(3):207-17.

\section{Publisher's Note}

Springer Nature remains neutral with regard to jurisdictional claims in published maps and institutional affiliations. 